ROCZNIKI HUMANISTYCZNE

Tom LXVI, zeszyt 8 - 2019

DOI: http://dx.doi.org/10.18290/rh.2019.67.8-10

ANNA DOLATA-ZARÓD

\title{
LA POLYFONCTIONNALITE DES CONNECTEURS : LA RELATION DE CONTRASTE DANS LES TEXTES JURIDIQUES COMPARABLES RELATIFS AU DOMAINE DE LA BOULANGERIE-PÂTISSERIE
}

\author{
MULTIFUNCTIONALITY OF CONNECTORS: \\ THE RELATION OF CONTRAST IN TEXTS COMPARABLES \\ ON THE EXAMPLE OF BAKERY-CONFECTIONERY DISCOURSE
}

Abstract

The aim of the analysis is to investigate how connectors contribute to the relations of contrast in the texts concerning one of the fields of handicraft, i.e. baking and confectionery. We will examine the characteristic features of selected connectors and compare these mechanisms in French and Polish texts. The first part of the study contains a short overview of the current research on connectors in French and Polish literature. In the second part, we present a comparable corpus including original French and Polish texts: collective labour agreements and statutes in the field of baking-confectionery. In the final, third part of the article, we conduct a comparative analysis of selected connectors showing the relation of contrast (French mais and Polish ale).

Key words: connectors ; relations ; contrast ; discourse.

\section{INTRODUCTION}

Les réglementations sont un ensemble de documents juridiques dont le but est de garantir les règles particulières du droit du travail applicable à un secteur donné. Lorsque l'on limite cette constatation strictement au domaine de la boulangerie-pâtisserie, on peut définir ces dispositions comme des

Dr ANNA DOLATA-ZARÓD - travaille à l'Institut des Langues Romanes et de la Traduction de l'Université de Silésie. Elle s'intéresse à la jurilinguistique et à la linguistique textuelle; adresse de correspondance : ul. Grota-Roweckiego 5, 41-205 Sosnowiec ; courriel : azarod@wp.pl. 
règles fixant les conditions de travail et du fonctionnement des artisans et des organisations artisanales. Le Code du travail français et polonais, détermine non seulement ses dispositions, mais aussi les lois et règlements applicables relatifs aux droits et obligations des employés et employeurs, ainsi que les dispositions des conventions collectives et d'autres accords comme statuts et règlements. Dans ce type de discours on remarque prédominance de la phrase déclarative constituant l'outil linguistique au moyen duquel la norme sera mise en oeuvre avec toute évidence. Cela est réalisé entre autres grâce aux connecteurs qui jouent un rôle de cohérence et de cohésion dans le texte.

Rossari (2000) qui traite la question de la relation entre connecteurs et relations de discours distingue trois grandes approches : conceptuelle, fonctionnelle et lexicale. La démarche conceptuelle s'appuie sur le repérage et la description de relations discursives de type logique ou intentionnel, le point de vue fonctionnel montre les liens de dépendance entre plusieurs segments du discours. L'approche lexicale s'appuie sur les indices linguistiques pour étudier les relations de discours qui permettent un accès au sens des relations de discours. Comme chacun des connecteurs véhicule sa propre relation de discours, nous proposons dans cet article une réflexion sur la présence de la relation de contraste dans le corpus spécialisé des textes relatifs à l'artisanat en polonais et en français. Notre étude vise à apporter quelques réflexions concernant l'analyse contrastive des connecteurs dans les textes français et polonais relatifs au domaine de la boulangeriepâtisserie : les conventions collectives et les statuts.

L'objectif de cette analyse est d'observer comment les connecteurs participent à la relation de contraste dans les textes relatifs au domaine de la boulangerie-pâtisserie, en d'autres termes, d'examiner ce que leurs caractéristiques intrinsèques confèrent à la création de ces types de relations et de comparer ces phénomènes dans les documents français et polonais. Premièrement, nous allons définir le cadre théorique. Pour ce faire, nous décrirons les traits caractéristiques de l'emploi des connecteurs et ensuite, nous discuterons des similitudes et des différences essentielles introduites par les connecteurs en français et en polonais. Deuxièmement, nous allons examiner le corpus français et polonais que nous avons assemblé. Notre corpus comparable est constitué de textes originaux français et polonais. Ce sont les textes relatifs au domaine de la boulangerie-pâtisserie: les conventions collectives et les statuts. Puis, nous allons examiner des connecteurs du discours français et polonais, en s'appuyant sur des exemples concrets pour voir enfin la relation de contraste. 


\section{LE CADRE THÉORIQUE}

Un connecteur a pour rôle d'indiquer le lien à établir entre deux segments de discours pour obtenir l'interprétation. Le lien établi est appelé relation de discours ou relation de cohérence. Depuis les travaux de Hobbs (1985), la notion de relation de discours a fait l'objet d'une littérature importante par exemple sur la distinction entre relations subordonnantes et coordonnantes (Polanyi 1985) ainsi que sur l'inventaire des relations et leur organisation hiérarchique (Grosz et Sidner 1986 ; Mann et Thompson 1988 ; Asher 1993). Parmi les travaux sur les connecteurs français il faut mentionner entre autres : Ducrot (1980, 1984), Anscombre \& Ducrot (1995), Moeschler (1985), Lane (1992), Rossari (2000), François \& le Querler (2000). Les études sur les connecteurs polonais englobent : Gizbert-Studnicki T. (2009), Grochowski (1984, 1995), Jadacka (1995a, 1995b, 1996, 2006), Labocha (2008, 2012), Lizisowa (2008, 2010, 2013), Malinowski (2006, 2008), Pawelec (2009), Wronkowska S. \& Zieliński M. (1993, 1997), Zieliński (2002, 2004).

Les connecteurs discursifs peuvent être considérés de manière générale comme des éléments particuliers de la langue dont la fonction est de lier deux segments textuels par une relation sémantique et de structurer le texte. L'existence de telles marques est généralement acceptée. Cependant, ils ont donné lieu à des définitions très diverses (Taboada 2006) ce qui résulte en des inventaires de connecteurs différents. Nous nous restreignons ici aux études qui lient les connecteurs et les relations discursives.

Cela signifie que seront considérés comme connecteurs des éléments liant des unités acceptées comme unités discursives. Ainsi, les connecteurs peuvent lier des propositions à l'intérieur des phrases mais aussi des phrases, ils participent donc à la segmentation en séparant les unités discursives intraphrastiques et à la structuration globale du texte. Vergas (1999) différencie dans sa description des connecteurs entre deux niveaux : celui de la microstructure du texte et celui de son contenu sémantique. Par conséquent, sur le plan formel les connecteurs « concernent la relation entre deux phrases, ils fonctionnent au niveau de la micro-organisation textuelle » et sur le plan sémantique ils « servent à indiquer explicitement des rapports entre les contenus des deux phrases, rapports qui peuvent être de type temporel (puis, alors), spatial (plus loin, dehors), argumentatif (toutefois, par contre), etc. »(Vergas 1999, 54). A ces deux niveaux, s'ajoute le niveau de la macro-organisation textuelle où les connecteurs peuvent signaler à niveau global des relations supra-phrastiques entre les parties d'un texte (Dridi 2017, 30). Selon Tremblay \& al. « Pour 
qu'un texte soit intelligible, il est nécessaire de pouvoir établir des relations sémantiques entre les phrases et les paragraphes » (Tremblay \& al. 1994, 54). De ce fait, les connecteurs se réalisent soit au niveau local entre deux propositions juxtaposées et reliées par leurs rapports sémantiques, soit au niveau global dans l'organisation de la structure du texte. De plus, les connecteurs discursifs doivent pouvoir déclencher une relation rhétorique. Dans ce cadre, Jayez et Rossari (1998) définissent les connecteurs pragmatiques comme des marqueurs discursifs particuliers, imposant une connexion sémantique entre propositions, fonctionnant comme des prédicats sur des arguments de différents types. Ces connecteurs sont de catégorie syntaxique variable : adverbes de phrase, conjonctions de subordination et prépositions. Jayez et Rossari (1998) distinguent les connecteurs de contraste des connecteurs d'opposition. Les premiers sont analysés comme des connecteurs typés (par contre) et les seconds comme des connecteurs inférentiels (pourtant). Les connecteurs typés prennent en argument des fragments d'information partageant un même type informationnel tandis que les connecteurs inférentiels fournissent des informations sur l'interprétation et ils indiquent quels types d'inférences doivent être combinées, et comment elles doivent l'être.

Nous nous appuyons sur la SDRT (Segmented Discourse Representation Theory) qui est une théorie de représentation du discours (Asher et Lascarides, 1993) dont l'un des objectifs est de mettre en évidence les mécanismes qui permettent aux locuteurs d'inférer des relations entre les segments d'un discours. Au départ, elle a été construite comme une extension de la DRT (Discourse Representation Theory, développée par Kamp, 1981) pour prendre en compte la complexité structurale d'un discours. Elle propose de représenter d'une part le contenu sémantique du discours, ou contenu de l'information (Information Content) dans des boîtes définies comme en DRT, et d'autre part, la macrostructure du discours ou emballage de l'information (Information Packaging) sous forme de graphes.

\section{LE CORPUS}

Selon Teubert (1996: 245) des « corpus comparables » sont des corpus en deux langues ou plus composés de façon identique ou similaire. Les textes qu'ils contiennent peuvent être classés selon une variété de traits intralinguistiques et extralinguistiques. Le domaine, par exemple, peut être une caractéristique pertinente pour la composition du corpus. La liste des critères 
sur lesquels s'appuie la comparabilité selon Teubert (1996) reste ouverte et n'est pas exhaustive : aux domaines on peut ajouter les thèmes, les genres textuels, la période, etc. Selon nous, un des critères essentiels pour la constitution d'un corpus comparable est celui du genre textuel, parce qu'il existe des genres comparables à travers les langues (p.ex. article scientifique, article de presse, roman) et parce que le genre subsume tout un ensemble de prescriptions normatives sur le plan de la textualité.

Notre corpus comparable est constitué de textes originaux français et polonais. Ce sont des textes juridiques relatifs au domaine de la boulangeriepâtisserie : les conventions collectives et les statuts. Ces documents précisent l'élément le plus important du droit du travail : le contrat de travail. Ils ont l'effet juridique.

Selon Cornu (2005 : 213) « est juridique tout message qui tend à l'établissement ou à l'application des normes de droit ». Dans ce discours qui n'est pas homogène, C. Bocquet (2008) propose trois types de textes juridiques : les textes normatifs (le discours du législateur), les textes des décisions qui appliquent ces normes (le discours du juge) et les textes qui exposent le contenu des règles de droit (le discours de la doctrine). Les conventions collectives et les statuts font partie du discours normatif qui recouvre le discours législatif et administratif comme ayant plusieurs points communs fonctionnels et stylistiques.

Comme le genre est un paramètre important de la construction du corpus parce qu'il rallie en effet le texte à une situation dans la réalité et dans les pratiques de rédaction et de lecture, nous présenterons quelques caractéristiques de la convention collective et des statuts. La convention collective correspond à des « accords conclus entre des organisations syndicales et les employeurs traitant des droits applicables aux salariés d'une entreprise ou d'une branche professionnelle » (Soignet 2003 : 78). La convention collective est un document rédigé entre employeurs et employés et visant à spécifier et à adapter les règles édictées par le code du travail selon la spécificité d'un secteur d'activité ou d'une profession particulière. Elle établit un second cadre législatif et permet d'ajuster les dispositions générales du Code du travail à un environnement géographique et professionnel donné. La nature collective de ce type de convention tire son caractère collectif de ses signataires, les syndicats « représentatifs » et les organisations reconnues par l'État dans leur secteur d'activité (Didry 2016, 1563).

Une convention collective doit contenir des éléments qui concernent aussi bien les salariés que les employeurs. Ainsi, l'accord écrit doit, entre autres, 
mentionner : la grille salariale, le mode de calcul des congés payés, la mutuelle, la prévoyance, les primes, le mode de calcul des indemnités en cas de rupture de contrat de travail, le régime appliqué lors des jours fériés, les préavis en cas de rupture de contrat de travail. Ces constatations sont valables pour les documents français et polonais. Il faut préciser qu'en Pologne, le type d'accords en question porte le nom de uktad zbiorowy pracy. Ainsi, le terme convention collective nationale est rendu comme krajowa umowa zbiorowa.

Le statut est un document dans lequel figurent d'une part ses éléments d'identité (titre, but et adresse du siège social) et d'autre part, l'ensemble des règles de fonctionnement qu'elle se fixe. Le statut constitue un contrat qui s'applique entre les membres de l'association et relèvent à ce titre et dans le domaine juridique, du droit des contrats ${ }^{1}$. Les statuts régissent le fonctionnement de l'association et les relations entre ses membres. Ils sont obligatoires pour l'accomplissement des formalités de déclaration de l'association. La rédaction des statuts permet aussi de clarifier et de formaliser ses buts et modes de fonctionnement Les mentions obligatoires du statut sont : titre, objet, adresse du siège social, conditions d'admission et catégories de membres, ressources et moyens d'action, instances décisionnelles (bureau, conseil d'administration, assemblée générale ordinaire et extraordinaire ou tout autre modèle de structuration choisi par l'association), répartition des pouvoirs et des tâches, modalité de désignation des élus et durée de leur mandat, procédures relatives aux modifications de statuts et à la dissolution, mode de convocation des assemblées générales ainsi que le quorum requis pour qu'elles se tiennent valablement et majorité requise lors des votes ${ }^{2}$.

Notre corpus a été consulté en ligne et il est composé de documents émanant d'un domaine spécialisé faisant appel à un langage spécialisé. Il est comparable au niveau du domaine (la boulangerie-pâtisserie) et au niveau du genre textuel (la convention collective et le statut) en présentant la même situation énonciative. Notre choix portant sur les textes juridiques relatifs au domaine de la boulangerie-pâtisserie tient au fait que nous postulons que ces textes sont pertinents car ils s'adressent à une communauté des experts et ils portent sur des cas d'échanges de nature argumentative. Comme le genre textuel est associé au type de discours et au domaine, nous remarquons que

\footnotetext{
${ }^{1}$ http://www.ddcs.paris.gouv.fr/SITE-DDCS/Engagement-citoyennete-et-vie-associative/Vie-as sociative/Creer-une-association-Centre-de-ressources-et-d-informations-pour-les-benevoles-CRIB/ Statuts-et-Reglement-Interieur (consulté le 02-04-2019).

${ }^{2}$ Ibidem (consulté le 02-04-2019).
} 
nos textes choisis partageant un même genre ont généralement une structure et un style propre.

\section{LES CONNECTEURS CHOISIS DE LA RELATION DE CONTRASTE}

Selon Mann et Thompson (1988) on peut caractériser sémantiquement la relation de contraste comme l'existence de deux segments présentant de nombreuses similarités, et aussi quelques différences, de manière à ce que l'accent puisse être mis à la fois sur la comparabilité et sur les différences entre les situations. La différence entre les segments peut se manifester de plusieurs façons : il peut s'agir d'une différence sémantique, que ce soit à un niveau lexical ou non lexical mais il peut s'agir aussi d'une différence pragmatique. Selon Busquets (2007), dans les termes de SDRT, la relation de contraste englobe plusieurs types d'oppositions. Premièrement, le cas où il y a un contraste (une dissemblance) entre deux états de choses qui partagent par ailleurs des similarités lexico-syntaxiques (une ressemblance) ; les deux faits sont comparés, mais sans qu'ils soient en relation de contradiction. Deuxièmement, le cas où le second membre réfute une attente qui émerge du premier membre (il y a une relation de cause à effet sousjacente). Troisièmement, les enchaînements négatifs prennent une valeur " rectificative », et il s'agit d'une construction assez différente des deux premières classes de structures.

Quant à la structure syntaxique des connecteurs, Ducrot (1980 : 99) explique trois positions du connecteur mais : Mais entre position $\mathrm{P}$ mais $\mathrm{Q}$, en tête de la proposition $\mathrm{Q}$ explicitée, mais $\mathrm{P}$, et en tête d'une expression interjective comme par exemple Mais moi j'aimerais bien apprendre le piano. Mais placé au début d'une phrase apparaît souvent dans une conversation et il sert à marquer une transition avec ce qui précède et met en relief ce qui va être dit. Vu nos genres textuels dans notre corpus comparable il n'y a pas de mais en position initiale ; nous analyserons le connecteur mais en position médiane.

Les expressions linguistiques qui servent (ou peuvent servir) à marquer la relation de contraste sont essentiellement des connecteurs, qui peuvent être (syntaxiquement) coordonnants (fr. mais, pourtant, cependant; pl. ale, jednak, jadnakże, choć) ou (syntaxiquement) subordonnants (fr. bien que, malgré que; pl. mimo że, mimo, aczkolwiek). Ces connecteurs sont tous considérés 
comme connecteurs de contraste dans la SDRT. Cependant, ils ne peuvent pas tous être regroupés sous la même relation de discours.

\section{LE CONNECTEUR FRANÇAIS MAIS ET POLONAIS $A L E$}

Le connecteur mais est un des connecteurs de la catégorie « oppositionconcession ». La fonction de mais est à la fois de relier et de mettre en opposition deux propositions selon le schéma « $\mathrm{P}$ mais $\mathrm{Q}$ », $\mathrm{Q}$ étant un « redressement argumentatif » par rapport au sujet de $\mathrm{P}$ : «tout discours [P mais $\mathrm{Q}]$ articule deux argumentations, [P donc $r$ ] et [Q donc non-r], de conclusions contraires » (Ducrot 1980). En analysant les deux types de mais : argumentatif et rectificatif on constate que le mais rectificatif sert à rectifier et le mais argumentatif introduit une proposition Q qui est orientée vers une conclusion non-r, opposée à une conclusion vers laquelle $\mathrm{P}$ pourrait conduire. Anscombre et Ducrot font une différenciation entre deux mais en français, le mais opérateur est lié à une "subordination sémantique ", et le mais connecteur est lié à une " coordination sémantique " (Anscombre, Ducrot 1977/1995: 23-40).

Ducrot (1980 : 94) de son côté, fournit une classification des diverses occurrences de mais dans son corpus. Il s'agit de : mais de transition illustré par « mais revenons à notre sujet", mais d'approbation " mais vous avec raison », mais d'addition : " non seulement mais encore », mais de renforcement : « on ne lui a donné rien à faire, mais ce qui s'appelle rien » et mais d'invitation: mais venez donc déjeuner.

Le trait le plus caractéristique du mais de réfutation est que la proposition qui le précède est à la forme négative. Il s'agit d'une négation explicite, exprimée dans l'exemple (1) par morphème ne pas. Ce mais apparaît entre deux segments d'une même phrase. La stratégie consiste à mentionner, dès le départ, la visée argumentative : « le salarié n'est pas à la disposition » et à introduire un énoncé « il peut vaquer librement à des occupations personnelles » qui justifie une telle visée. On voit qu'entre les segments de la phrase existe un thème contrastif consistant à être ou ne pas être à la disposition de l'employeur.

(1) Le travailleur de nuit bénéficie d'une pause d'une durée minimale de 20 minutes pour toute période de travail effectif atteignant 6 heures. Si durant cette pause le salarié n'est pas à la disposition de l'employeur mais peut vaquer libre- 
ment à des occupations personnelles, ce temps de pause n'est pas considéré comme du travail effectif et n'est pas rémunéré [A -Article 23].

On observe la même stratégie dans l'exemple suivant (2). Ici, le deuxième segment il peut atteindre exceptionnellement 10 heures s'oppose à une inférence qu'on pourrait tirer du premier segment la durée maximale quotidienne $d u$ travail effectuée par un travailleur de nuit ne peut excéder 8 heures. Donc, plus le thème est contrastif, meilleure sera la relation. Le degré de contraste est défini en termes de degrés de différence entre les propositions qui marquent les thèmes des nœuds de la structure sémantique.

(2) La durée maximale quotidienne du travail effectuée par un travailleur de nuit ne peut excéder 8 heures mais peut atteindre exceptionnellement 10 heures [A Article 23].

Il faut souligner la nuance existante entre les environnements droit et gauche du connecteur. Dans ces cas-là, mais est toujours précédé d'une négation polémique c'est-à-dire qu'elle " introduit un acte de réfutation qui s'oppose à un autre acte d'assertion antérieur ou virtuel » (Moeschler 1989 : 63). Le premier argument de l'exemple (2) constitue la partie thématique de l'énoncé ; c'est le deuxième argument à la forme affirmative qui fait l'objet de l'effet juridique et transmet l'information nouvelle.

Eggs (1994) distingue entre autres un mais compensatoire (substituable par «en revanche»: «Pierre n'est pas intelligent mais/en revanche il est charmant») et un mais contrastif (substituable par par contre : "Pierre aime les chocolats mais/par contre Jacques aime les biscuits »). Dans l'exemple (3), un mais articule deux thèmes différents ayant deux expansions rhématiques différentes et selon la typologie de Eggs, on y retrouve un mais contrastif qui joue le rôle argumentatif.

(3) Le contrôle immédiat du travail n'est pas toujours possible, mais les répercussions des non-conformités se manifestent rapidement. Le temps d'adaptation ne peut excéder 2 mois [A - Article 4].

Dans l'exemple suivant (4), au niveau syntaxique, le connecteur mais relie deux énoncés indépendants dont la structure doit étre obligatoirement explicitée.

(4) Pour répondre aux besoins actuels et futurs des entreprises des industries alimentaires en matière de qualifications, mais aussi dans la perspective de répondre 
à l'évolution des métiers et au renforcement des compétences des salariés s'inscrivant dans la gestion des parcours professionnels, afin de favoriser leur maintien et leur évolution dans l'emploi, tant interne qu'externe, les signataires du présent accord confirment leur volonté de promouvoir la formation professionnelle [A - Article 6.3].

En analysant cet exemple nous ne pouvons pas dire que les deux segments reliés par mais aient un rapport d'opposition, au contraire le connecteur mais intensifié ici par aussi exprime une valeur de renforcement.

Dans l'exemple (5), le connecteur mais coordonne un énoncé négatif à un énoncé positif.

(5) Le sauveteur secouriste du travail ne doit pas limiter son action à la gestion des incidents, mais doit également devenir un véritable acteur de prévention [A Article 86].

Ici, il s'agît des obligations du sauveteur secouriste du travail qui doit s'occuper de la gestion des incidents et de la prévention. Le rapport de contraste ici est plutôt faible et indirecte, et grâce à l'opérateur également on apporte un argument additionnel pour la conclusion et on exprime une valeur additive. On constate que cette stratégie d'employer du connecteur mais avec aussi en (4) ou également en (5) s'établit le plus souvent lorsque mais apparaît entre des éléments de phrase ou entre deux propositions liées à un même sujet (le sauveteur secouriste du travail).

Analysons maintenant les exemples du corpus comparable polonais. Le connecteur ale marque une opposition entre deux propositions en apportant une restriction (Kieliszczyk 1999, 2012), (Dutka 1990, 1992, 1993), Grochowski (1984, 1996), Jadacka (1995a, 2006), Lizisowa (2008), Malinowski (2006, 2008) comme dans l'exemple (6) :

(6) Władze mają prawo dokooptowania na miejsce ustępujących i nieaktywnych członków w czasie kadencji nowych członków władz, ale w ilości nie przekraczającej 1/3 składu pochodzącego z wyboru [B - § 20].

Dans l'exemple (7), les segments reliés par le connecteur mais ont en commun d'être relatifs aux cotisations, et se distinguent sur la hauteur de ces derniers.

(7) Członek nadzwyczajny ma obowiązek: 1) popierać działalność SRP, 2) opłacać zadeklarowane składki miesięcznie, ale w wysokości nie mniejszej niż uchwalonej przez Walne Zebranie Członków [B - §15]. 
Dans l'exemple qui suit (8), le connecteur ale sert aussi à renforcer un avis par l'emploi de także et à exprimer l'addition.

(8) Do obowiązków Zarządu Głównego należy [...] powoływanie i rozwiązywanie Kół regionalnych oraz nadzorowanie ich działalności, ale także organizowanie zebrań zwyczajnych członków nie należących do Kół regionalnych, niezależnie od obowiązku wynikającego z pkt. 3) [A - §24].

Dans l'exemple (9) un premier argument $\mathrm{P}$ et un deuxième argument $\mathrm{Q}$ ne sont pas en relation de contradiction. Nous remarquons ici un apport d'une restriction sur ce qui vient d'être dit ( $w$ możliwie najkrótszym terminie, ale nie później niż w ciagu czternastu dni).

(9) Na wniosek Komisji Rewizyjnej posiedzenie Zarządu powinno odbyć się w możliwie najkrótszym terminie, ale nie później niż w ciągu czternastu dni od dnia złożenia wniosku $[\mathrm{C}-\S 35]$.

\section{CONCLUSION}

Nous avons présenté quelques remarques concernant des connecteurs exprimant la relation de contraste dans le discours de la boulangerie-pâtisserie. Nous avons analysé des documents relatifs à l'artisanat en polonais et en français en prenant en considération les conventions collectives et les statuts. Ces documents constituent l'un des éléments du droit du travail. C'est pourquoi nos conclusions ont recours d'une part au niveau de la micro-organisation textuelle et d'autre part au plan sémantique pour déterminer explicitement des rapports entre les contenus des deux phrases.

Dans notre corpus, la relation de contraste est réalisée notamment par le connecteur français mais et polonais ale. Dans les deux langues il se trouve dans la position médiane. D'après les exemples cités et dans les termes de SDRT, nous constatons que la relation de contraste englobe en français le mais d'opposition jouant le rôle argumentatif, le mais à valeur rectificative, additive ou de renforcement. Nous avons aussi montré que les deux segments reliés par mais ayant un rapport d'opposition renforcé par aussi ou également expriment une valeur additive. Notre analyse prouve que le connecteur ale en polonais exprime le plus souvent l'addition ou la restriction. Nous n'avons pas retrouvé dans les textes polonais des propositions avec des arguments formulés en négation suivis de ale. C'est la différence la plus 
importante qui résulte de notre comparaison des textes juridiques comparables en français et en polonais.

\section{BIBLIOGRAPHIE}

Amsili Pascal \& Winterstein Grégoire, 2015, «Quelques combinaisons de connecteurs discursifs », Verbum XXXVII (2), 323-342.

Anscombre Jean-Claude \& Ducrot Oswald, 1977, « Deux mais en français », [in :] Lingua 43, 23-40.

Anscombre Jean-Claude \& Ducrot Oswald, 1983/1995, L'argumentation dans la langue, Brussels, Pierre Mardaga.

Asher Nicholas, 1993, Reference to Abstract Objects in Discourse, Amsterdam, Academic Publishers.

Asher Nicholas \& Lascarides Alex, 1998, "The Semantics and Pragmatics of Presupposition », Journal of Semantics vol. 15, is. 3, 239-300.

Bocquet Claude, 2008, La traduction juridique; fondement et méthode, Bruxelles, De Boeck.

Busquets Joan, 2007, «Discourse Contrast : Types an Tokens », [in :] Aurnague M., Korta K. \& Larrazabal J.M. [eds.], Language, Representation and Reasoning. Memorial Volume to Isabel Gómez Txurruka, Bilbao, University of Basque Country Press, 103-123.

Cornu Gérard, 2005, Linguistique juridique, $3^{\mathrm{e}}$ édition, Paris, Montchrestien.

Dridi Mohammed, 2017, «Types et fonctions des connecteurs dans les articles scientifiques de 'la Revue des bioressources' ", [in :] Les Revues de l'Université Kasdi Merbah Ouargla 28, 25-34.

Didry Claude, 2016, «La production juridique de la convention collective. La loi du 4 mars $1919 »$ Annales. Histoire, Sciences Sociales, 2001, no 6 (56 année), 1253-1282.

Ducrot Oswald, 1980, Les Mots du discours, Collection Le sens commun, Paris, Les éditions du Minuit.

Ducrot Oswald, 1984, Le dire et le dit, Paris, Les éditions de minuit.

Dutka Anna, 1990, «Propozycja opisu morfemu ale w ujęciu teorii argumentacji », Acta Philologica 21, 39-49.

Dutka Anna, 1992, « A propos des morphèmes proches de ale 'mais' en polonais dans l'approche argumentative », [in :] Kwartalnik Neofilologiczny 39, no 1, 27- 44.

Dutka Anna, 1993, «Les connecteurs argumentatifs en polonais », [in :] Dittmar N. \& Reich A. [éds.], Modality in Language Acquisition. Modalité et acquisition des langues, Berlin, De Gruyter, 97-109.

Eggs Ekkehard, 1994, Grammaire du discours argumentatif, Paris, Kimé.

François Jacques \& Le Querler Nicole, 2000, Syntaxe et sémantique, Caen: Presses universitaires de Caen.

Gizbert-Studnicki Tomasz, 2009, «Postulat jasności i zrozumiałości tekstów prawnych a dostęp do prawa », [in :] Mróz A., Niewiadomski A. \& Pawelec M. [éds.], Prawo i język, Warszawa, Zakład Graficzny Uniwersytetu Warszawskiego, 9-18.

Grochowski Maciej [éd.], 1995, Wyrażenia funkcyjne w systemie i tekście, Toruń, Wydawnictwo UMK.

Grochowski Maciej, 1986, Polskie partykuty. 'Sktadnia, semantyka, leksykografia', Wrocław, Zakład Narodowy im. Ossolińskich, Wydawnictwo Polskiej Akademii Nauk. 
Grochowski Maciej, 1996, «O partykułach jako wykładnikach nawiązania. Analiza wyrażenia wręcz », [in :] Grochowski M. [éd.], Anafora w strukturze tekstu, Warszawa, Wydawnictwo Energeia, 97-104.

Grochowski Maciej, 2004, "Czy istnieje tekst poza gatunkiem? », [in :] Ostaszewska D. [éd.], Gatunki mowy i ich ewolucja, t. 2, Tekst a gatunek, Katowice, Gnome, 20-28.

Grochowski Maciej, Karolak Stanisław \& Topolińska Zuzanna, 1984, Gramatyka wspótczesnego języka polskiego. Sktadnia, Warszawa, PWN.

Grosz Barbara J., Sidner Candance L., 1986, «Attention, intentions, and the structure of discourse », Journal Computational Linguistics, vol. 12, issue 3, 175-204.

Hadermann Pascale, Pierrard Michel \& Van Raemdonck Dan, 2006, «Les marqueurs d'identité: subordonnants, coordonnants ou corrélateurs ? », Faits de Langues, 28, 133-144.

Hobbs Jerry, 1985, On the Coherence and Structure of Discourse (Research Report 85-37). Stanford, CA: CSLI.

Jadacka Hanna, 1995a, «O związkach gramatycznych między wyrazami w zdaniu », Biuletyn Rady Legislacyjnej 4.

Jadacka Hanna, 1995b, « Od czego zależy zrozumiałość tekstu », Biuletyn Rady Legislacyjnej 6.

Jadacka Hanna, 1996, « O zdaniach za długich i za krótkich », Biuletyn Rady Legislacyjnej 2.

Jadacka Hanna, 2006, Poradnik językowy dla prawników, Warszawa: Semper.

Jayez Jacques \& Rossari Corinne, 1998, «Discourse relations versus discourse merker relations », [in :] Proceedings of the ACL'98 workshop on discourse relations and discourse merkers, 72-78.

Kamp Hans, 1981, «A theory of truth and semantic representation », [in :] Groenendijk J.A.G., Janssen T.M.V., Stokhof M.B.J. [éds.], Formal methods in the Study of Language, Amsterdam, Mathematisch Centrum, 277-322.

Kieliszczyk Anna, 1992, «Francuskie „mais” i niektóre jego odpowiedniki w języku polskim », [in :] Acta Philologica 22, 47-57.

Kieliszczyk Anna, 1999, « Le fonctionnement argumentatif des connecteurs d'opposition en français et en polonais », Acta Philologica 26, 42-52.

Kieliszczyk Anna, 2012, «L'analyse argumentative des connecteurs d'opposition dans la traduction du français en polonais ", [in :] Vasilescu A.et al. [éds.], Challenges in Translation: Space, Culture and Linguistic Relativity, New York, Addleton Academic Press, 387-394.

Labocha Janina, 1996, « Tekst, wypowiedź, dyskurs », [in :] Gajda S. \& Balowski M. [éds.], Styl a tekst. Materiały międzynarodowej konferencji naukowej, Opole, Wydawnictwo Uniwersytetu Opolskiego, 49-53.

Labocha Janina, 2008, Tekst, wypowiedź, dyskurs w procesie komunikacji językowej, Kraków, Wydawnictwo Uniwersytetu Jagiellońskiego.

Labocha Janina, 2012, «Płaszczyzny opisu tekstu », [in :] Pachowicz M. \& Choińska K. [éds.], Mundus verbi. In honorem Sophiae Cygal-Krupa, Tarnów, Wydawnictwo Państwowej Wyższej Szkoły Zawodowej w Tarnowie, 218-226.

Lane Philippe, 1992, La périphérie du texte, Paris, Nathan.

Lizisowa Maria Teresa, 2008, « Semantyka a struktura tekstu prawnego », [in :] Szczepankowska I. [éds.], Styl a semantyka, Białystok, Wydawnictwo Uniwersytetu w Białymstoku, 381-400.

Lizisowa Maria Teresa, 2010, «Tekst prawny w teorii znaku i teorii komunikowania », [in :] Mróz A., Niewiadomski A., \& Pawelec M. [éds.], Prawo, język, etyka, Warszawa, Zakład Graficzny Uniwersytetu Warszawskiego, 51-66. 
Lizisowa Maria Teresa, 2013, « Metody badania języka prawnego wobec ontologicznej natury prawa », Comparative Legilinguistics 14, 19-43.

Malinowski Andrzej, 2006, Polski język prawny. Wybrane zagadnienia, Warszawa, Wydawnictwo Prawnicze LexisNexis.

Malinowski Andrzej, 2008, Redagowanie tekstu prawnego. Wybrane wskazania logiczno-językowe, Warszawa, Wydawnictwo Prawnicze LexisNexis.

Mann William C. \& Thompson Sandra A., 1988, « Rhetorical Structure Theory: Toward a functional theory of text organization », Text vol. 8(3), 243-281.

Moeschler Jacques, 1985/1990, Argumentation et conversation : éléments pour une analyse pragmatique du discours, Paris, Didier.

Moeschler Jacques, 1989, Modélisation du dialogue. Représentation de l'inférence argumentative, Paris, Hermès.

Moeschler Jacques, 2002, "Connecteurs, encodage conceptuel et encodage procédural. Nouveaux regards sur les mots du discours ", Cahiers de linguistique française 24, 265-292.

Pawelec Radosław, 2009, «Poprawność w związkach zgody, rządu i przynależności; szyk wyrazów i skróty składniowe » [in :] Malinowski A. \& Bałaban A. [éds.], Zarys metodyki pracy legislatora: ustawy, akty wykonawcze, prawo miejscowe, Warszawa, Wydawnictwo Prawnicze LexisNexis, 371-407.

Polanyi Livia, 1985, «Conversational storytelling », [in :] Van Dijk T.A. [éds.], Discourse and Dialogue, vol. 3 of Handbook of Discourse Analysis 4. London: Academic Press, s. ???.

Rossari Corinne, 2000, Connecteurs et relations de discours : des liens entre cognition et signification, Nancy, Presses Universitaires de Nancy.

Rossari Corinne, 1999, "Les relations de discours avec ou sans connecteur », Cahiers de linguistique française 21, 181-192.

Soignet Michel, 2003, Le français juridique, Paris, Hachette Fle.

Taboada Maite \& Mann William C., 2006, "Applications of Rhetorical Structure Theory » Discourse Studies vol. 8(4), 567-588.

Teubert Wolfgang, 1996, « Comparable or Parallel Corpora?», International Journal of Lexicography vol. 9, is. 3, 238-264.

Tremblay Robert \& al., 1994, Le texte argumentatif et les marqueurs de relation, $\mathrm{BN}$ de Québec, Université Laval.

Wróblewski Bronisław, 1948, Język prawny i prawniczy, Kraków, Polska Akademia Umiejętności.

Vergas Claude, 1999, Grammaire pour enseigner, tome 1, L'énoncé, Le texte, La phrase, Paris, Armand Colin.

Wronkowska Sławomira \& Zieliński Maciej, 1993, Problemy i zasady redagowania tekstów prawnych, Warszawa, Urząd Rady Ministrów.

Wronkowska Sławomira \& Zieliński Maciej, 1997, Zasady techniki prawodawczej: komentarz, Warszawa, Wydawnictwo Sejmowe.

Zieliński Maciej, 2002, Wykładnia prawa. Zasady. Reguly. Wskazówki, Warszawa, Wydawnictwo Prawnicze LexisNexis.

Zieliński Maciej, 2004, « Język prawny, język administracyjny, język urzędowy », [in :] Malinowska E. [éd.], Język - prawo - spoleczeństwo, Opole, Wydawnictwo Uniwersytetu Opolskiego. 


\title{
CORPUS
}

A: Convention collective nationale des activités industrielles de boulangerie et pâtisserie du 13 juillet 1993. Mise à jour par avenant $\mathrm{n}^{\circ} 10 \mathrm{du} 11$ octobre 2011.

https://www.legifrance.gouv.fr/affichIDCC.do?idConvention=KALICONT000005635691, consulté le 02-04-2019.

B : Statut Stowarzyszenia Rzemieślników Piekarstwa Rzeczypospolitej Polskiej, http://mistrz branzy.pl/public/uzytkownicy/statuty/180-statut.pdf, consulté le 02-04-2019.

C : http://cech.gdansk.pl/sites/cech.gdansk.pl/files/1461239579/Statut\%20Cechu\%20tekst\%20jed nolity.pdf, consulté le 02-04-2019.

\section{LA POLYFONCTIONNALITE DES CONNECTEURS : LA RELATION DE CONTRASTE DANS LES TEXTES JURIDIQUES COMPARABLES RELATIFS AU DOMAINE DE LA BOULANGERIE-PÂTISSERIE}

\section{Résumé}

L'objectif de cette analyse est d'observer comment les connecteurs participent à la relation de contraste dans les textes relatifs au domaine de la boulangerie-pâtisserie, en d'autres termes, d'examiner ce que leurs caractéristiques intrinsèques confèrent à la création de ces types de relations et de comparer ces phénomènes dans les documents français et polonais. Premièrement, nous allons décrire brièvement l'état des recherches sur les connecteurs dans la littérature française et polonaise. Deuxièmement, nous allons nous pencher sur notre corpus comparable constitué de textes originaux français et polonais : les conventions collectives et les statuts. Puis, nous allons mener l'analyse comparative des connecteurs choisi concernant de la relation de contraste (français mais et polonais ale).

Mots-clés : connecteurs; relations; contraste; discours.

\author{
WIELOFUNKCYJNOŚĆ KONEKTORÓW: RELACJA KONTRASTU \\ W PORÓWNYWALNYCH TEKSTACH PRAWNICZYCH \\ NA PRZYKŁADZIE DYSKURSU PIEKARSTWA-CUKIERNICTWA
}

Streszczenie

Celem niniejszej analizy jest sprawdzenie, w jaki sposób konektory przyczyniają się do powstawania relacji kontrastu $\mathrm{w}$ tekstach dotyczących jednej $\mathrm{z}$ dziedzin rękodzieła to jest piekarstwa-cukiernictwa. Zbadamy cechy charakterystyczne wybranych konektorów i porównamy te mechanizmy w tekstach francuskich i polskich. Pierwsza część pracy zawiera krótki przegląd dotychczasowych badań nad konektorami w literaturze francuskiej i polskiej. W części drugiej przedstawimy korpus porównywalny obejmujący oryginalne teksty francuskie i polskie: zbiorowe układy pracy i statuty z dziedziny piekarstwa-cukiernictwa. W ostatniej, trzeciej, części pracy przeprowadzimy analizę porównawczą wybranych konektorów tworzących relację kontrastu (francuskie mais i polskie ale).

Slowa kluczowe: konektory; relacje; kontrast; dyskurs. 